\title{
A Novel Approach to Control the Frequency and Voltage of Microgrids in Islanding Operation
}

\author{
Mohammad Reza Ebrahimi, Mohammad Naser Hashemnia, Mehdi Ehsan, and Amir Abbaszadeh
}

\begin{abstract}
In this paper, electrical parameters of a microgrid containing distributed generation wind and fuel cell units are controlled in islanding mode operation. Islanding operation refers to isolation of a part of power system including distributed generation from the grid having continuous and independent operation. This has negative impacts on the isolated network including voltage and frequency dependence on load. These impacts and their solutions are studied in this paper. A proper controller using power electronics converters has been proposed. In order to verify the validity of the proposed control approach, it has been implemented and simulated on a 13-bus IEEE distribution power system containing a combination of distributed generation wind and fuel cell units.
\end{abstract}

Index Terms-Frequency controd, inverter, islanding operation, microgrids, voltage control.

\section{INTRODUCTION}

Restructuring of power system, environmental concerns regarding concentrated power plants, instantaneous changes of electrical energy price and fast technological development of distributed generation (DG) units have all made distributed generation ever increasing. A distribution network to which a ditributed generation system is connected is called a microgrid[1].

A microgrid appears when an electrical region can control its voltage and frequency and supply its load automatically and independently from the grid. Existing rules in electrical grids don't still let independent operation of microgrids due to several reasons such as safety of operators, stability, etc. This means that distributed generation units should be isolated from the grid as soon as possible [2] (Of course, there are special cases where microgrids are allowed to operate).

The early power grids can be considered as the first distributed generation units as there were only DC networks and transmission of energy to long distances had therefore much energy loss. After appearance of power transformers, DC networks were gradually replaced by AC networks. Large power plants were founded at suitable places and energy was transferred long distances. The new trend towards DG has been due to several reasons as:

- Technological development in DG units

- Increased need to build new transmission lines

- Reliable energy demand

- Economical concerns

- Environmental concerns

Manuscript received June 25, 2012; revised August 23, 2012.

The authors are with the Center of Excellence in power System Management and Control (CEPSMC) of Sharif University of Technology.
The two last cases are the most important reasons.

\section{Challenges, Difficulties AND DRAwbaCKS OF MICROGENERATION IN ISLANDING OPERATION}

Microgrid operation in islanding mode will be subject to several problems some of which are highlighted below:

1) Voltage oscillations: Using DG units may result to problems regarding control of bus voltages, especially in smaller microgrids and at situations where there is peak load and minimum generation.

2) Frequency oscillations: Any unbalance between production and consumption will result to a change in frequency at an electrical network. This is mostly important in small grids as the change in frequency will be more severe.

3) Power quality issues: A high amount of generation at DG units is delivered to the grid via power electronic devices. They are a source of harmonics due to their nonlinearity and the switching phenomenon. Therefore, part of their generated harmonics is injected to the grid. Suitable filters should be designed and used to mitigate this effect.

\section{Microgrids CONTROL In ISLANDING ModE}

Most micro sources are not suitable for direct connection to low voltage grid; hence $\mathrm{DC} / \mathrm{AC}$ or $\mathrm{AC} / \mathrm{DC} / \mathrm{AC}$ converters are used as the medium between the suppliers and low voltage grid. Control of these converters is the approach for frequency and voltage control of microgrids in islanding mode. Two kinds of control can be adopted to operate an inverter device [3]:

\section{A. Inverter Control Based on $P-Q$ Mode}

In this method, supplier's output active and reactive powers are controlled based on predetermined set points. This control mode is in association with control of both the inverter and the prime mover if physically applicable. The inverter injects the active power it receives at its input to the grid. This kind of control can be implemented in photovoltaic units. The inverter's injected reactive power is set to the predetermined value which is assigned locally or via microgrid control center. The P-Q control mode can be realized with current control technique; the amplitude and phase of inverter current are controlled in such a way that the required active and reactive powers are achieved.

The inverter's injected reactive power is set to the pre-specified value which is assigned locally or via microgrid control center. The P-Q control mode can be realized with 
current control technique; the amplitude and phase of inverter current are controlled in such a way that the required active and reactive powers are achieved [4].

\section{B. Inverter Control Based on Voltage Control Mode}

In this method, the inverter feeds the load based on voltage and frequency reference values using a certain control method. The voltage source inverter in the microgrid actually plays the role of a synchronous generator.

Considering such a VSI operating in parallel with an AC system with angular frequency $\omega_{\text {grid }}$, the active output power of the VSI is automatically defined by the well known frequency droop characteristic as in Fig. 1. In this figure, the droop characteristic has been drawn for two parallel inverters Indices 1 and 2 refer to first and second converters respectively. The output power of each inverter can be varied by changing its idle frequency.

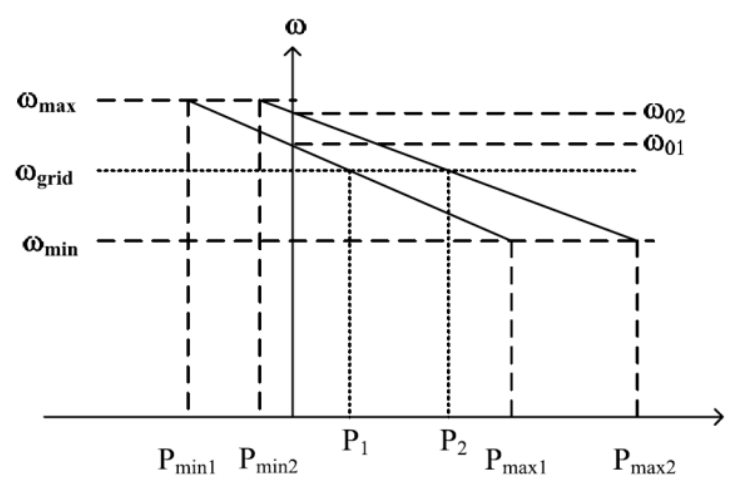

Fig. 1. Frequency droop characteristic.

If the main AC system is lost, there will be a change in output power of each inverter according to its droop characteristic and the network frequency drifts towards a new one. Active power is shared between the inverters according to their droop characteristic. Similarly, the relation between voltage and reactive power can be expressed using the concept of voltage droop. The voltage droop characteristic has been depicted in Fig. 2.

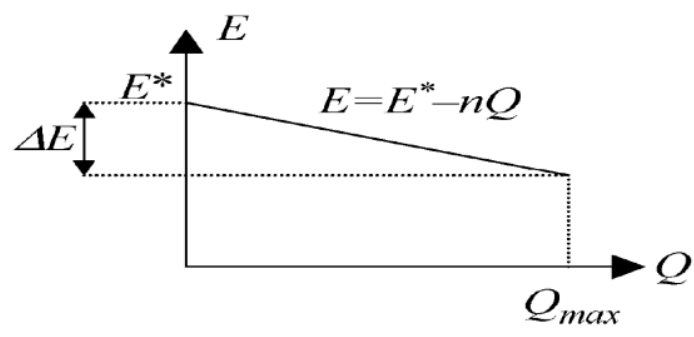

Fig. 2. Voltage droop characteristic.

A balanced three-phase model including both the voltage droop and frequency droop characteristics has been shown in Fig. 3 [5]. The decoupling transfer functions are due to the delay in measurement of active and reactive powers. The active and reactive powers determine the output frequency and voltage of the inverter according to the frequency and voltage droop characteristics respectively. A phase feed-forward control with gain kff has been added to the system for stability purposes [5].

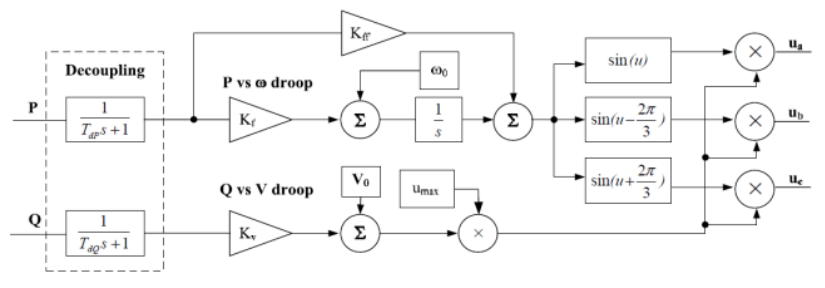

Fig. 3. VSI three phase control model.

If the main power supply (the MV network) is available, all the inverters can be operated in the PQ mode, because a voltage and frequency reference is available. However if the MV network is lost all the inverters will shut down because there will not be a voltage reference within the MG and it will not be possible to obtain an exact balance between load and generation. This means that a general frequency control strategy should be followed in order to operate the MG in islanded mode [2].

\section{PROPOSED CONTROL METHOD}

\section{A. Method Fundamentals}

It is apparent that an islanded network consists of loads and sources. One of the souces is usually taken as voltage and frequency controller and the other energy sources produce their active and reactive powers in a range defined by the control center.

The controller should maintain the voltage in the reference bus together with the frequency of the whole network. Desired amplitude and phase of the voltage are achieved by applying reference values $V_{d_{-} r e f}$ and $V_{q_{-} \text {ref }}$.

Two PI controllers are used to maintain the voltage at reference value. Phase angle signal is generated from the reference frequency of PWM by an oscillator.

\section{B. Controller Design}

Three phase voltages at the point of island connection to the grid $V_{a b c}^{p c c}$ (pcc refers to point of common coupling) are measured and the values of $\omega$ and $\theta$ are then extracted using a PLL block.

The produced currents of DG are then measured and then transformed to dq components $\left(I_{d}\right.$ and $\left.I_{q}\right)$ using the angle $\theta$ extracted by the PLL. They are compared with their reference values ( $I_{d-r e f}$ and $\left.I_{q-r e f}\right)$. The outputs of the comparators are then fed to PI controllers the outputs of which will be dq reference voltage components $\left(V_{d-r e f}\right.$ and $\left.V_{q-r e f}\right)$. These voltages are sent to $d q-m, \delta$ block in ${ }_{\mathrm{o}}$ rder to compute the amplitude $(m)$ and phase angle $({ } \delta)$ of the bus voltage as follows:

$$
\begin{gathered}
m=\sqrt{V_{d-r e f}^{2}+V_{q-r e f}^{2}} \\
\delta=\tan ^{-1}\left(\frac{V_{q-r e f}}{V_{d-r e f}}\right)
\end{gathered}
$$


These values are then fed to the PWM block in order to build signals to control the power electronic devices. As a result, there will be a three-phase voltage with amplitude $m$ and phase angle $\delta$ at the inverter output. The stages denoted are shown in Fig. 4.

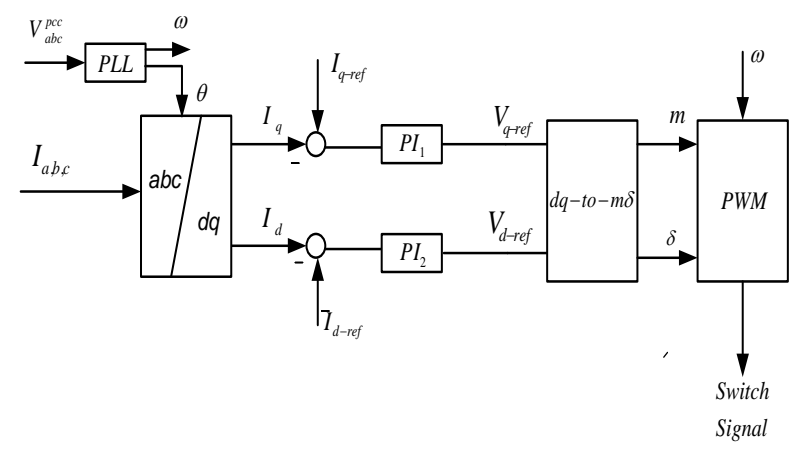

Fig. 4. Controller design using the proposed approach.

The abc bus voltages can also be extracted directly instead of calculating the amplitude and phase angle ( $m$ and $\delta$ ).

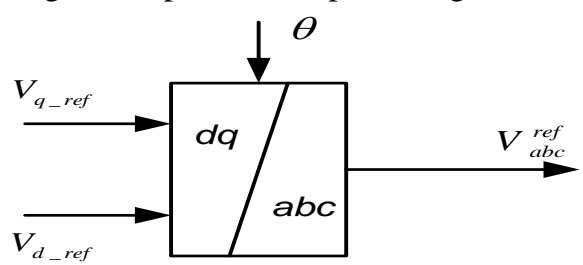

Fig. 5. Direct voltage synthesis using its dq components.

As it is observed, the dq voltages are transformed to three-phase abc voltages using the measured angle.

\section{CASE Study}

A standard distribution network has been chosen and the controllers are implemented on it in order to validate the proposed control approach. The studied network in this paper is the IEEE modified 13-bus network where the DG units installed are a combination of wind and fuel cell units.

All the data regarding this network including the lines, DG units and distributed loads are given in [6]. In the following simulations, the internal dynamics of the fuel cell unit are neglected and the fuel cell has simply been represented by its Thevenin's equivalent circuit. The nominal active power of the fuel cell unit is 1.7 megawatts. It has been supposed that this unit works at a worst power factor of 0.8 . Therefore, its maximum reactive power can be computed as following: $P_{D G 1}=1.7 \mathrm{MW}$

$$
Q_{D G 1}=P_{D G 1} * \tan \Phi=1.275 M V A R
$$

The frequency and nominal voltage of the IEEE 13-bus network are $60 \mathrm{~Hz}$ and $4.16 \mathrm{Kv}$ with $\pm 1 \%$ and $\pm 5 \%$ tolerance respectively. The loads of this network are:

$$
P+j Q=3.466 M W+j 2.102 M V A R
$$

The sum of nominal powers of generators should be more than total loads in order for the microgrid to be able to operate in islanding mode. It has been supposed that a wind farm with nominal power $P+j Q=2.3 M W+j 1.7 M V A R$ has been connected to bus No.680. Therefore, the total generation of the microgrid will be equal to:

$$
\begin{aligned}
& P_{g r i d}+j Q_{g r i d}=P_{d g 1}+P_{d g 2}+j\left(Q_{d g 1}+Q_{d g 2}\right)= \\
& 4 M W+j 2.975 M V A R
\end{aligned}
$$

It is observed that the total generation capacity of the microgrid is more than its maximum load and it is therefore capable of operation in islanding mode.

The single line diagram of the implemented network inPscad/EMTDC is shown in Fig. 6.

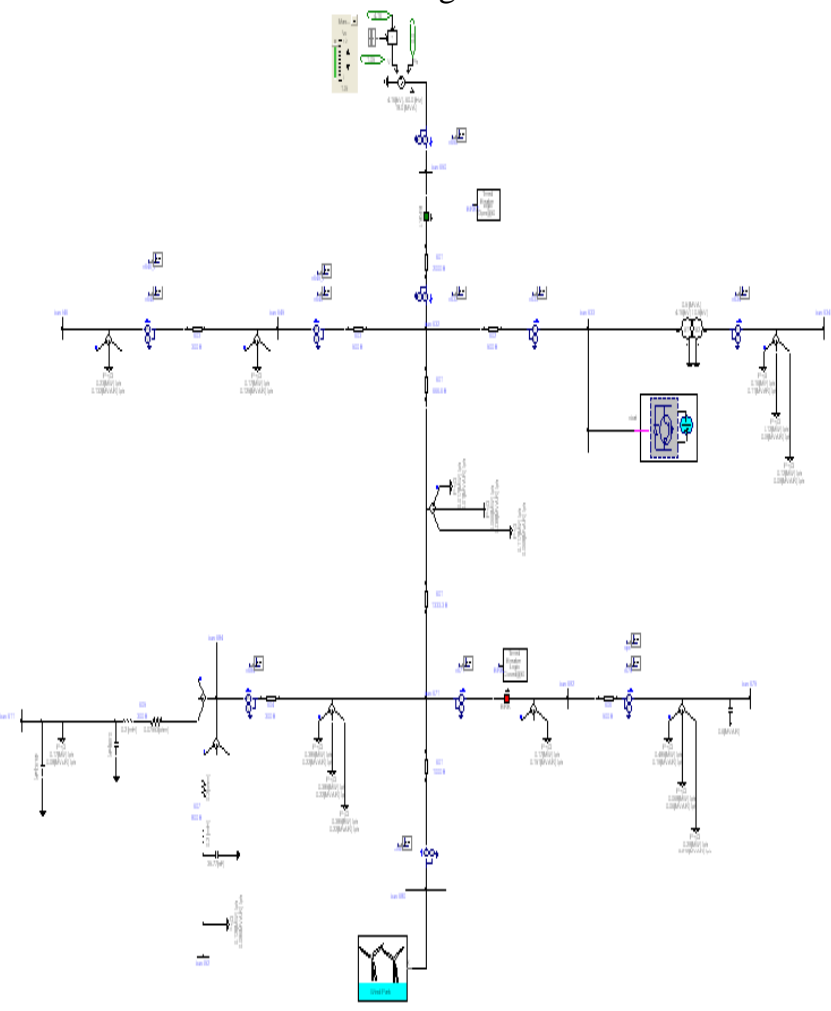

Fig. 6. Single line diagram of the implemented network.

\section{Steady State PeRformance StUdy}

In order to study the steady state performance of the proposed control method, line-line terminal voltage of the generator $\left(E_{a b 1}\right)$, currents of various parts of DG unit, input voltage of the inverter before and after filtering and variation of bus voltages are simulated. All the simulations are done for 5 seconds. Line-line terminal voltage of the wind generator before and after filtering has been shown in Fig 7. As it is observed, the filter prevents high frequency current harmonics from entering the generator side. The source of current harmonics is the power electronic converter.

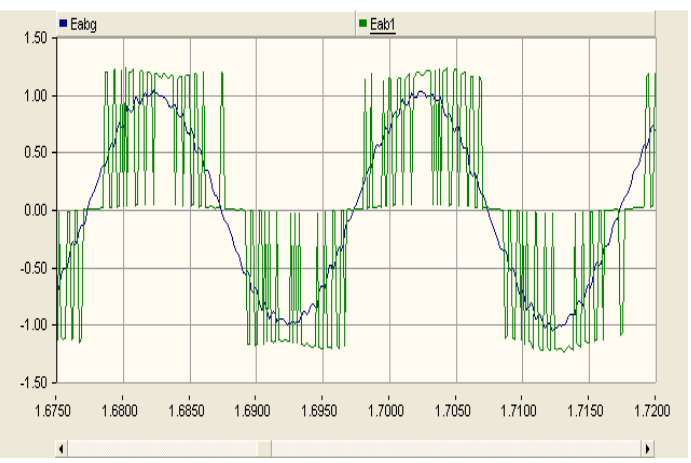

Fig. 7. Terminal voltage of the wind generator before and after filtering 


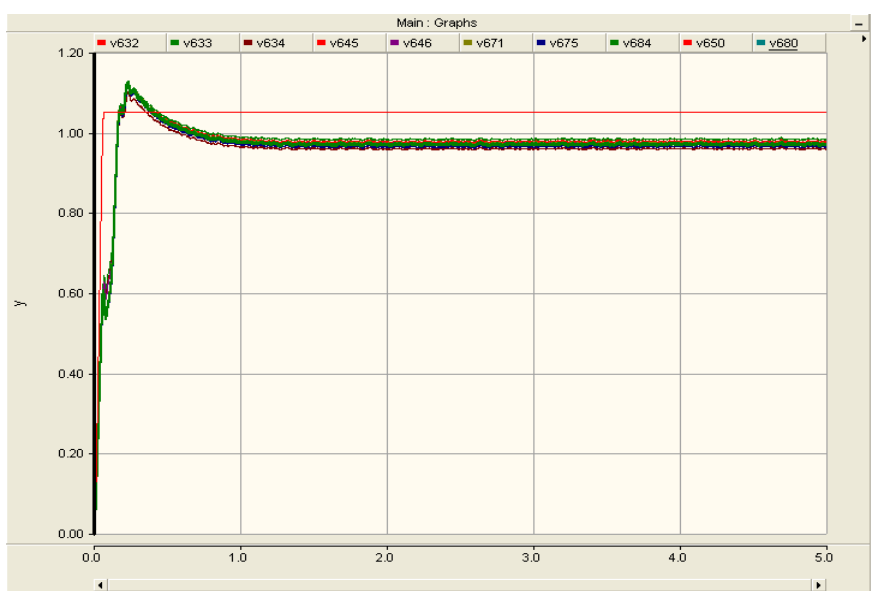

Fig. 8. Voltages of buses

Voltages of all buses are depicted in Fig 8. It is obvious that all the voltages reach a steady state value after the transients due to islanding decay.

Currents of various parts of DG unit, namely generator phase current (blue), transformer current (black), rectifier current (red) and inverter current (green) are shown in Fig 9. It is visible that the harmonic oscillations are much more in the inverter and rectifier than the generator and transformer. Rms output voltage of the inverter before (blue) and after (green) filtering are shown in Fig 10. It is observed that the voltage before filtering has more oscillations with higher overshoot. Instantaneous output voltage of one phase of the inverter before (blue) and after (red) filtering are shown in Fig 11. It is again apparent that the voltage before filtering has more oscillations with higher overshoot.

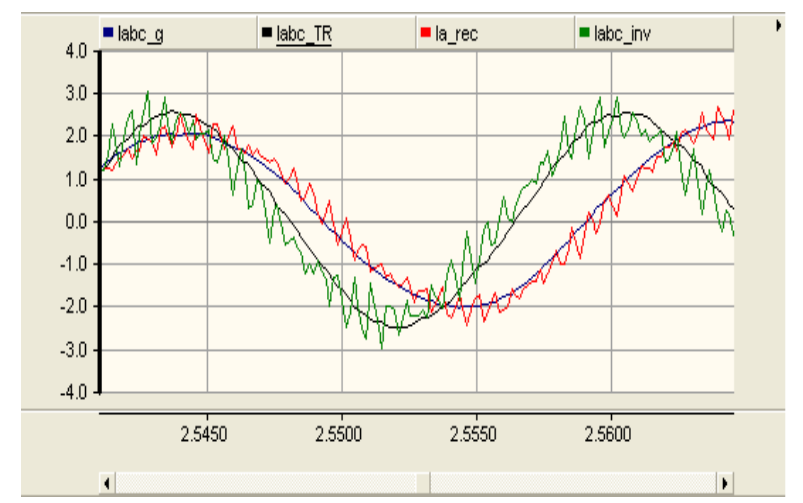

Fig. 9. Currents of various parts of DG unit.

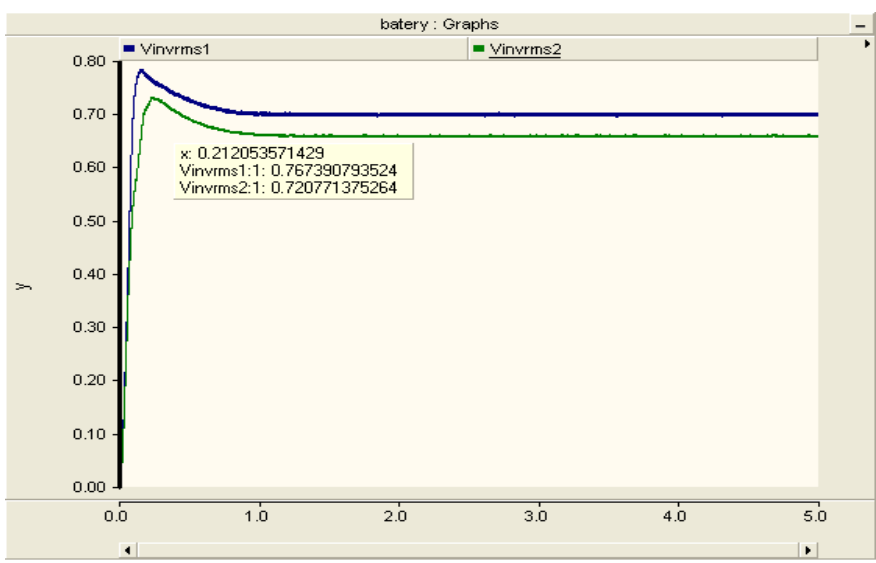

Fig. 10. Rms output voltage of the inverter before (blue) and after (green) filtering.

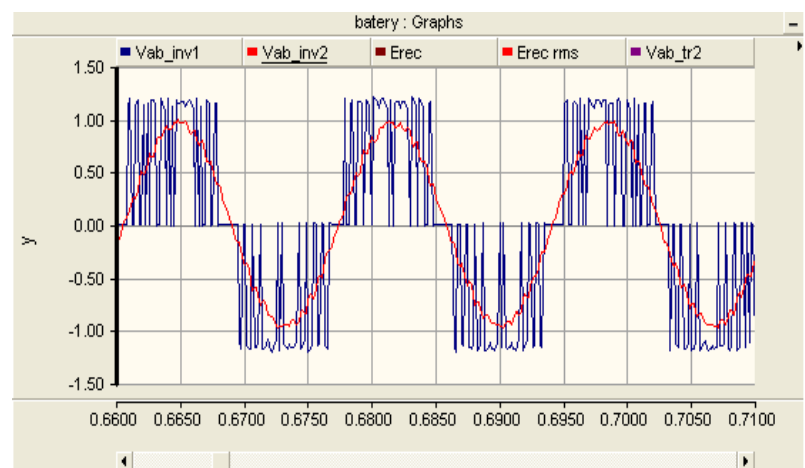

Fig. 11. Instantenous output voltage of the inverter before (blue) and after (red) filtering.

\section{Performance Study In CASe of Fault}

A single phase short circuit is applied to the first phase of bus No. 671 at $\mathrm{t}=3 \mathrm{sec}$ for 3 cycles and then it is cleared. Variation of voltage of different buses is shown in Fig 12. It is visible that the fault causes the voltage of several buses to reduce to $0.77 \mathrm{pu}$ and there is an overshoot after fault clearance which returns to its normal value due to proper operation of the controller. It is obvious in Fig 13 that the terminal voltage of the generator is also affected by the short circuit, but the controller has been able to return it to its normal value. It is visible that the voltage before filtering has higher oscillations and overshoot.

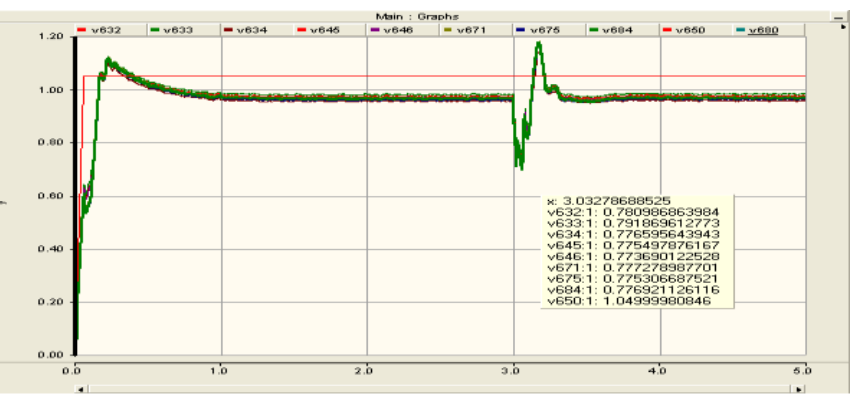

Fig. 12. Variation of bus voltages due to single phase short circuit.

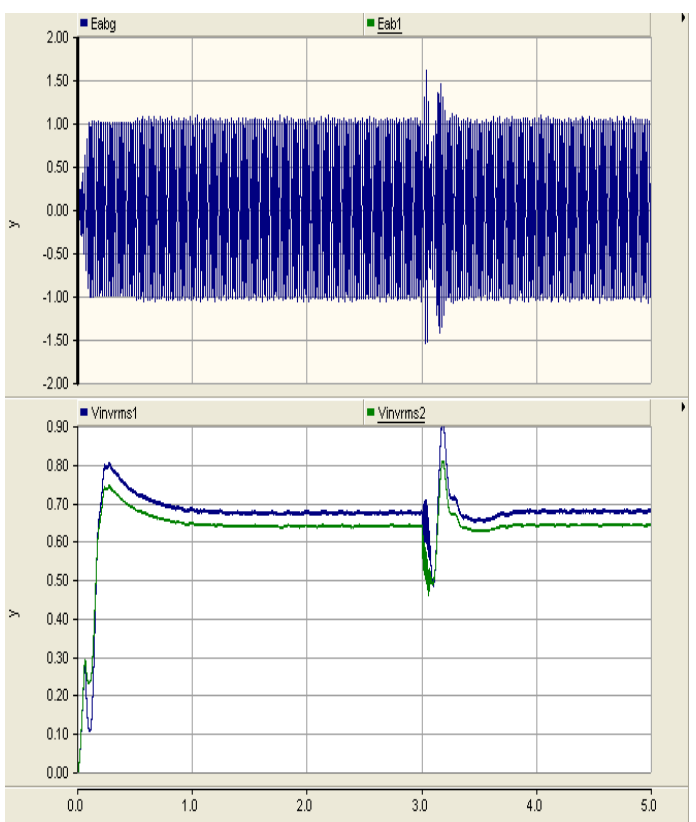

Fig. 13. Variation of instantenous and rms values of generator terminal voltage before (blue) and after (green) filtering. 


\section{CONCLUSION}

Distributed generation units can be connected to the grid via rotating electric machines or power electronics converters. On the other hand, they can operate in grid connected or islanded mode. In microgrids, a small change in load or generation can lead to variation of frequency and voltage due to low production capacity. Therefore, controllers used in these networks should have low delay and high reliability with high compatibility. Power electronic converters have almost all the aforementioned specifications, so they have been used to keep frequency and voltage constant in this paper. Power electronic converters can support voltage angle and amplitude stability by controlling their output active and reactive powers, respectively. In this study a control pattern using park transformation has been used for frequency and voltage control of microgrids. Its performance has been evaluated in 13-bus standard network. Simulation results show the method is successful in voltage and frequency control with varying network parameters.

Park transformation is used to transform the 3- phase voltages to direct and quadrature axis components; proper control is applied in this space and then the inverse transformation is used to attain the real 3-phase quantities. Moreover, use of suitable filters for mitigation of harmonics caused by inverter controllers due to their nonlinearity has been studied.

\section{ACKNOWLEDGMENT}

This work is supported by the Center of Excellence in Power System Management \& Control (CEPSMC) of Sharif University of Technology.

\section{REFERENCES}

[1] J. A. Peças Lopes et al., "Management of microgrids," JIEEC 2003, Bilbao, pp. 28-29, October 2003.

[2] A. Peças Lopes, C. L. Moreira, and F. O. Resende, "Microgrids black start and islanded operation," 15th PSCC, Liege, pp. 22-26, August 200.

[3] Barsali, M. Ceraolo, P. Pelacchi, and D. Poli, "Control techniques of dispersed generators to improve the continuity of electricity supply," Trans. of PES Winter Meeting 2002, IEEE, vol. 2, pp. 789-794, 27-31 Jan. 2002.

[4] H. Akagi, Y. Kanazawa, and A. Nabae, "Instantaneous reactive power compensators comprising switching devices without energy storage components," IEEE Transactions on Industry Applications, vol. IA-20, no. 3, pp. 625-630, May/June 1984.
[5] J. A. Engler, "Regelung von batteriestromrichtern in modularen und erweiterbaren inselnetzen," Dissertation. de, Berlim, May 2002, ISBN 3-89825-439-9.

[6] Distribution Test Feeder Working Group, IEEE PES Distribution System Analysis Subcommittee. [Online]. Available: http://www.ewh.ieee.org/soc/pes/dsacom/testfeeders/index.html.

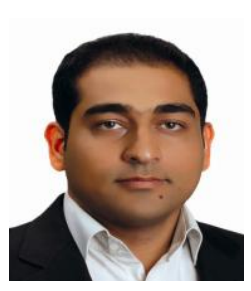

Mohammad Reza Ebrahimi was born in Tehran, Iran in 1983. He received his B.Sc. on 2006 from ferdowsi University of Mashhad(UM), Mashhad, Iran and M.Sc. on 2009 from Sharif University of Technology (SUT), Both in Electrical Engineering. Currently he is a faculty member in EE Department of Sama Technical and Vocational College Islamic Azad University, Tehran Branch. His research interests are Power \& Energy System Analysis, Power Systems Restructuring and Deregulation, Power Markets, Reliability of Power Systems and Distributed Generation.

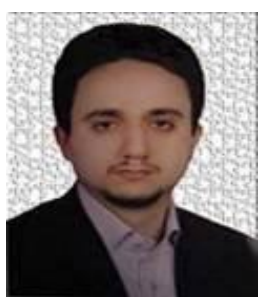

Mohammad Naser Hashemnia was born in Mashad, Iran, in 1984. He received his B.S. in Electrical Power Engineering from Ferdowsi University, Mashad, Iran, in 2006 and his M.S. in Electrical Power Engineering from the University of Tehran, Tehran, Iran, in 2008. He is currently a Ph.D. student at Sharif University of Technology, Iran, where he is working on the modeling and control of Brushless Doubly Fed Machines (BDFMs). His main interests include the analysis and simulation of electric machines and advanced control methods for electrical drives.

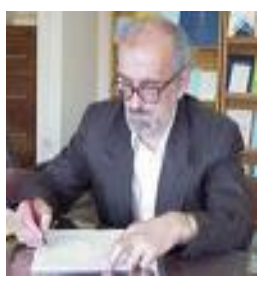

Mehdi Ehsan received B.Sc. \& M.Sc. in Electrical Engineering from Technical College of Tehran University 1963, PhD \& DIC from Imperial College University of London in 1976. Since then He has been with EE Dept of Sharif University of Technology in different responsibilities. He has an extended cooperation with research centers in Iran and also abroad. Currently he is a professor in EE Department of Sharif University. His research interests are Power system Simulation, Dynamic \& Transient Stability, Application of Expert Systems in Identification, Operation, and Planning \& Control of power system.

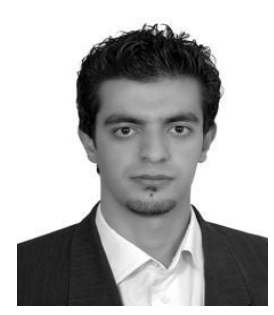

Amir Abbaszadeh was born in tehran in the islamic republic of iran, on 1985. he graduated from the shahid bahonar university of kerman in 2008 and received his m.sc. degree from science and research branch of azad university (srbiau), tehran, iran in 2011. in 2009 he joined the iran grid management company (igmc) as a dispatcher. his main field of interests includes the power system operation, power system dynamics and application of intelligent methods in power systems 